\title{
Tableau des symboles utilisés
}

\begin{tabular}{|l|l|}
\hline $\mathbf{a}, \mathbf{b}, \mathbf{c}$ & Vecteurs de base de la maille directe \\
\hline$a, b, c$ & Longueurs des vecteurs de base de la maille directe \\
\hline $\mathbf{a}^{*}, \mathbf{b}^{*}, \mathbf{c}^{*}$ & Vecteurs de base de la maille réciproque \\
\hline$a^{*}, b^{*}, c^{*}$ & Longueur des vecteurs de base de la maille réciproque \\
\hline$v_{0}$ & Volume de la maille directe \\
\hline$v_{0}^{*}$ & Volume de la maille réciproque \\
\hline$[u v w]$ & Rangée parallèle au vecteur $u \mathbf{a}+v \mathbf{b}+w \mathbf{c}$ \\
\hline$\langle u v w\rangle$ & $\begin{array}{l}\text { Ensemble des directions équivalentes à la direction } \\
\text { du groupe ponctuel cristallin }\end{array}$ \\
\hline$(h k l)$ & Famille des plans réticulaires d'indices de Miller $h, k$ et $l$ \\
\hline$(h k i l)$ & $\begin{array}{l}\text { Famille des plans réticulaires d'indices de Miller } h, k \text { et } l \\
\text { pour un réseau hexagonal }\end{array}$ \\
\hline$\{h k l\}$ & $\begin{array}{l}\text { Ensemble des plans réticulaires }(h k l) \text { équivalents par les } \\
\text { opérations de symétrie du groupe ponctuel cristallin }\end{array}$ \\
\hline$\{h k i l\}$ & $\begin{array}{l}\text { Ensemble des plans réticulaires }(h k i l) \text { équivalents par les } \\
\text { opérations de symétrie du groupe ponctuel cristallin pour } \\
\text { un réseau hexagonal }\end{array}$ \\
\hline$\left\{\begin{array}{l}\text { Distance entre deux plans réticulaires }(h k l) \text { voisins ou } \\
\text { distance réticulaire de la famille }(h k l)\end{array}\right.$ \\
\hline$d_{h k l}$ & Vecteurs de base d'un référentiel orthonormé \\
\hline $\mathbf{e}_{1}, \mathbf{e}_{2}, \mathbf{e}_{3}$ & $\begin{array}{l}\text { Matrice dont } M_{i j} \text { est le coefficient situé sur la } i \text { ème ligne } \\
\text { et } j \text { ème colonne. Elle est notée } M\end{array}$ \\
\hline$\left\{M_{i j}\right\}$ & $\begin{array}{l}\text { Matrice de passage d'un référentiel orthonormé } \\
\text { à un autre }\end{array}$ \\
\hline$\left\{a_{i j}\right\}=A$ & Vecteur polaire \\
\hline $\mathbf{V}$ & Vecteur axial \\
\hline
\end{tabular}




\begin{tabular}{|c|c|}
\hline$[T]$ & Tenseur \\
\hline$T_{i j}$ & $\begin{array}{l}\text { Composantes, dans un système d'axes orthonormés } \\
\text { donné, d'un tenseur }[T] \text { de rang } 2 \text {. La matrice associée } \\
\text { est notée } T\end{array}$ \\
\hline$T_{i j k}$ & $\begin{array}{l}\text { Composantes, dans un système d'axes orthonormés } \\
\text { donné, d'un tenseur de rang } 3\end{array}$ \\
\hline$e_{i j}$ & Composantes du tenseur $[e]$ des gradients de déplacement \\
\hline$[S], S_{i j}$ & Tenseur des déformations et ses composantes \\
\hline$[T], T_{i j}$ & Tenseur des contraintes et ses composantes \\
\hline$S_{\alpha}, T_{\alpha}$ & $\begin{array}{l}\text { Notation contractée de Voigt des tenseurs }[S] \text { et }[T] \text {. } \\
\alpha \text { varie de } 1 \text { à } 6 \text { et les composantes de ces tenseurs } \\
\text { forment alors des vecteurs colonne à } 6 \text { lignes }\end{array}$ \\
\hline$[s], s_{i j k l}$ & Tenseur d'élasticité et ses composantes \\
\hline$[c], c_{i j k l}$ & Tenseur de rigidité et ses composantes \\
\hline$\Theta$ & Température \\
\hline $\mathcal{S}$ & Entropie \\
\hline $\mathbf{E}$ & Champ électrique \\
\hline $\mathbf{D}$ & Induction \\
\hline $\mathbf{P}$ & Polarisation \\
\hline$[\varepsilon], \varepsilon_{i j}$ & $\begin{array}{l}\text { Tenseur de permittivité relative (ou de constante diélec- } \\
\text { trique) et ses composantes }\end{array}$ \\
\hline$\varepsilon_{0}$ & Permittivité (ou constante diélectrique) du vide \\
\hline$[\eta], \eta_{i j}$ & Tenseur d'imperméabilité électrique et ses composantes \\
\hline$\delta_{i j}$ & Symbole de Kronecker \\
\hline$\delta_{i j k}$ & Tenseur des permutations ou symbole de Lévi-Civita \\
\hline$[G], G_{i j}$ & Tenseur de gyration et ses composantes \\
\hline$[d], d_{i j k}$ & Tenseur de piézoélectricité et ses composantes \\
\hline$[r], r_{i j k}$ & $\begin{array}{l}\text { Tenseur électro-optique linéaire ou de Pockels et ses com- } \\
\text { posantes }\end{array}$ \\
\hline$[z], z_{i j k l}$ & $\begin{array}{l}\text { Tenseur électro-optique quadratique ou de Kerr et ses } \\
\text { composantes }\end{array}$ \\
\hline$[p], p_{i j k l}$ & Tenseur élasto-optique et ses composantes \\
\hline$[\pi], \pi_{i j k l}$ & Tenseur piézo-optique et ses composantes \\
\hline
\end{tabular}

